\title{
VALIDATION OF A HIGH FREQUENCY RADIO FREQUENCY IDENTIFICATION (HF RFID) SYSTEM FOR REGISTERING FEEDING PATTERNS OF GROWING-FINISHING PIGS
}

Maselyne, J., Saeys, W., De Ketelaere, B., Mertens, K., Vangeyte, J., Hessel, E.F., Millet, S., and Van Nuffel, A., 2014. Validation of a High Frequency Radio Frequency Identification (HF RFID) system for registering feeding patterns of growing-finishing pigs. Computers and Electronics in Agriculture 102, 10-18, doi:10.1016/j.compag.2013.12.015.

Jarissa Maselyne ${ }^{a, b}$, Wouter Saeys ${ }^{a}$, Bart De Ketelaere ${ }^{a}$, Kristof Mertens ${ }^{a}$, Jürgen Vangeyte $^{\mathrm{b}}$, Engel F. Hessel ${ }^{\mathrm{c}}$, Sam Millet ${ }^{\mathrm{d}}$, Annelies Van Nuffel ${ }^{\mathrm{b}, *}$

${ }^{a}$ Division Mechatronics, Biostatistics and Sensors (MeBioS), Department of Biosystems, Katholieke Universiteit Leuven, Kasteelpark Arenberg 30 bus 2456, 3001 Heverlee, Belgium

${ }^{b}$ Technology and Food Science Unit - Agricultural Engineering research area, Institute for Agricultural and Fisheries Research (ILVO), Burg. van Gansberghelaan 115 bus 1, 9820 Merelbeke, Belgium

'Division of Process Engineering, Department of Animal Science, Faculty of Agricultural Science, Georg-August-University of Goettingen, Universitätsstraße 7, 49377 Vechta, Germany

${ }^{d}$ Animal Sciences Unit, Institute for Agricultural and Fisheries Research (ILVO), Scheldeweg 68, 9090 Melle, Belgium

*Corresponding author: annelies.vannuffel@ilvo.vlaanderen.be, tel.: +32 927227 56, fax: +32 9272 2801

E-mail adresses: jarissa.maselyne@ilvo.vlaanderen.be (J. Maselyne),wouter.saeys@biw.kuleuven.be (W. Saeys), bart.deketelaere@biw.kuleuven.be (B.De Ketelaere), kristof.mertens@porphyrio.com (K. Mertens), jurgen.vangeyte@ilvo.vlaanderen.be (J. Vangeyte), earkena@gwdg.de (E. Hessel), sam.millet@ilvo.vlaanderen.be (S. Millet), annelies.vannuffel@ilvo.vlaanderen.be (A.Van Nuffel).

\section{Highlights:}

- A HF RFID system was validated for registering feeding pigs.

- The system was used in simulated commercial farm conditions.

- Specificity for individual pigs with one or two tags was always above $85 \%$.

- Sensitivity varied for individual pigs, tags and different time windows used.

- A sensitivity of $88.58 \%$ and a specificity of $98.34 \%$ can be reached 
Automated monitoring of the feeding patterns of growing-finishing pigs would allow detecting problems with individual pigs or groups of pigs and thus improving health, welfare and productivity of the farm. In this chapter a High Frequency Radio Frequency Identification (HF RFID) system was validated for its suitability to register individual pigs' feeding patterns at a round trough in a grouphousing context. High Frequency RFID antennas were installed above the troughs of a commercially available type of round feeder to identify feeding pigs fitted with one or two passive RFID tags on their ears. A multiplexer was used to connect multiple antennas to a single reader. During $11.5 \mathrm{~h}$, video observations of 20 focal pigs (equipped with two tags) at an age of 16 weeks were performed to validate the system.

A large variation in feeding patterns of the 20 focal pigs was found. Correlation between the number of registrations per pig and the feeding duration on video was low $\left(R^{2}=0.53\right)$ mainly due to four pigs with specific feeding behaviour (with the four pigs excluded: $\mathrm{R}^{2}=0.88$ ). The RFID registrations of the 20 focal pigs - with irregular time gaps between them - were compared with instantaneous video samples using several time window sizes around the video sample. The specificity for individual pigs with one or two tags was always above $85 \%$, but sensitivity varied for individual pigs, tags and with different time windows used. A quantitative comparison between the use of one or two tags per pig was made based on a receiver operating characteristic (ROC) curve. For two tags per pig a sensitivity of $88.58 \%$ and a specificity of $98.34 \%$ can be reached with a time window size of 9 s. For one tag per pig, sensitivity is only above $85 \%$ at a time window of size $31 \mathrm{~s}$. Of the total number of RFID registrations $77.11 \%$ occurred during feeding visits, and $92.23 \%$ occurred during or within $10 \mathrm{~s}$ of feeding visits on video.

This system showed good potential for measuring feeding patterns of growing-finishing pigs in commercial pig houses, for research purposes, or to detect potential problems with pigs by signalling changes in the registered feeding patterns.

Keywords: growing-finishing pigs, radio frequency identification, feeding pattern, validation

\section{INTRODUCTION}

With today's increasing herd sizes and increasing work load for pig farmers, visual contact and individual monitoring of animals has declined. This has created the need for automated monitoring of the animals (Frost et al., 1997). Automatic measurements of environmental parameters such as temperature and relative humidity are common practice. However, animal based measurements such as behavioural analyses can provide a better view of the animals' state and well-being. Alterations in feeding patterns are considered to be one of the first warning signs of health-, welfareand productivity problems in growing-finishing pigs (Hart, 1988; Weary et al., 2009). This makes that automatic registration of pigs' feeding patterns could support pig farmers in their daily management routine (Wathes et al., 2008). As feeding patterns can differ amongst pigs in the same pen, even in homogenous groups, automatic registration shows the most potential when applied to individual pigs (Brown-Brandl et al., 2013; Hessel and Van den Weghe, 2011).

Radio Frequency Identification (RFID) systems for animal registration have been used to measure feeding patterns. These systems are usually validated by comparing the RFID registered behaviour 
with video observations. In this way, the feeding pattern of dairy cows (DeVries et al., 2003; Chapinal et al., 2007), beef cattle (Schwartzkopf-Genswein et al., 1999; Brown-Brandl and Eigenberg, 2011; Mendes et al., 2011) and growing-finishing pigs (Reiners et al., 2009; Brown-Brandl and Eigenberg, 2011) registered with a Low Frequency (LF) RFID system has been validated.

Computerized feeding stations have been used to measure pigs' feeding patterns. Cornou et al. (2008) used data from electronic sow feeders to determine the individual feeding rank of sows. For growing-finishing pigs, electronic feed stations have been developed and used to log time and duration of feed visits (Hyun et al., 1997; Bruininx et al., 2001a; Bruininx et al., 2001b; Hyun and Ellis, 2002). These systems often also provide the possibility to measure or control the feed intake of each individual pig. Nevertheless, these feeders are only accessible to one pig at a time and there is often only one feeder in a pen, contrary to most commercial situations. These special circumstances may lead to higher competition around the feeder and the pigs may therefore adapt their feeding patterns accordingly (Nielsen et al., 1996; Gonyou and Lou, 2000). Therefore, it would be better to use more commercial feeders which can be accessed by multiple pigs. However, this would require identification of several individual pigs at the same time.

Despite the recent advances in the field of automated video tracking of pigs in group housing (Lind et al., 2005; Ahrendt et al., 2011), the most suitable method to automatically identify individual animals remains RFID (Artmann, 1999; Eradus and Jansen, 1999; Ruiz-Garcia and Lunadei, 2011). Radio Frequency Identification is a collection of techniques for contactless transfer of data between a datacarrying device and a reader using (electro)magnetic fields (Finkenzeller, 2010). The international standards that regulate the use of RFID for animals apply to passive LF systems with an operating frequency of $134.2 \mathrm{kHz}$ (ISO 11784 \& 11785) (Artmann, 1999; Finkenzeller, 2010).

While most commercial feeders can be accessed by multiple pigs at the same time, the currentlyavailable LF RFID systems are not capable of reading multiple tags at the same time. Brown-Brandl et al. (2013) solved this problem by installing one LF antenna per feeding access point in an industrial rectangular feeder and achieved good results in determining individual animal feeding patterns. For many other types of commercial feeders where the feeding points are not physically separated it is, however, not possible to install one antenna per feeding access point. Moreover, use of one antenna per multiple-access feeding point would also reduce the complexity and cost.

Using RFID systems with higher frequencies could be the answer to this challenge. Such systems often incorporate anti-collision algorithms to avoid losing data when multiple tags are in read-range. Using passive High Frequency (HF) RFID with an operating frequency of $13.56 \mathrm{MHz}$, feeding piglets were successfully identified in a semi-commercial group-housed situation (Reiners et al., 2009; Hessel and Van den Weghe, 2011). A higher frequency means higher data transmission rates and more resistance to electromagnetic interference, but also higher disturbance from metal or water in the neighbourhood (Chawla and Ha, 2007; Ruiz-Garcia and Lunadei, 2011). However, High Frequency (HF) RFID, as opposed to Ultra High Frequency (UHF) RFID, still penetrates water and body tissue well. Since both LF and HF RFID use the same data transfer technique (inductive coupling), read ranges and costs of the tags can be similar, but vary with size, memory, housing and extra features of the tags.

The aim of this study was to validate the effectiveness of a newly developed HF RFID system using multiplexers in detecting feeding pigs at a commercial-type feeder, by (1) searching for a correlation 
between number of RFID registrations and feeding duration, (2) calculating the performance of the system on group and animal level and using one or two tags per pig.

\section{MATERIALS AND METHODS}

\subsection{ANIMALS AND HOUSING}

The study took place at the experimental farm of the Institute for Agricultural and Fisheries Research (ILVO, Melle, Belgium). The pigs were housed in an automatically ventilated barn divided into four identical pens. Each pen measured $4.3 \mathrm{~m}$ by $9 \mathrm{~m}$ with approximately $40 \%$ slatted concrete floor and $60 \%$ solid concrete lying area. Water was supplied via four nipple drinkers in each pen. Dry pelleted feed was automatically supplied using 8 Swing MIDI feeders (Big Dutchman Pig Equipment GmbH, Vechta, Germany). The pigs were fed a commercially available feed with $9.3 \mathrm{MJ}$ net energy, $15.50 \%$ crude protein and $0.92 \%$ lysine ad libitum.

In total, 236 growing-finishing pigs (Hybrid sow $x$ Piétrain boar) with an age of $10 \pm 1$ weeks (mean \pm standard deviation (SD)) were randomly assigned to the four pens to achieve groups with equal numbers of barrows and gilts and a similar mean weight and variation. Each pen contained 30 barrows and 29 gilts. The average weight of the animals on arrival was $19.3 \pm 2.0 \mathrm{~kg}$ (mean \pm SD). The pigs remained in the stable until they reached an average slaughter weight between 110 and $120 \mathrm{~kg}$ at day 157 in the fattening period. During the fattening period, the average daily gain of the pigs was $0.639 \mathrm{~kg} /$ day. Experiments were approved by ILVO's Ethical Committee and were in accordance with EU Directive 2010/63/EU for animal experiments.

\subsection{MEASUREMENTS}

RFID INFRASTRUCTURE

To measure the feeding patterns, the pigs were equipped with RFID tags on their ears. The tags used were IN Tag 300 I-Code SLI tags (ISO 15693, HID Global Corporation, California, USA) with a diameter of $30.0 \mathrm{~mm}$, a thickness of $2.5 \mathrm{~mm}$ and an enlarged hole of $7.8 \mathrm{~mm}$. These RFID tags were fitted onto the pig using the pin of standard pig ear tags and had approximately the same diameter as the ear tags, as illustrated in Figure 1. All 236 pigs in the stable were equipped with a HF tag clipped onto an ear tag. In one pen, all 59 pigs were equipped with an additional HF tag in their second ear. Of the 295 tags present in the stable during the fattening round, 10 were lost from the ears and replaced. All other tags remained functional during the entire fattening period.

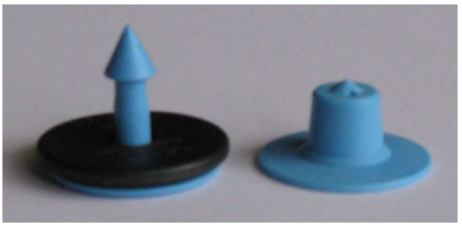

Figure 1: High Frequency RFID tag (black) attached to standard pig ear tag.

A HF RFID system at the trough registered the presence of these tags when they came within range of the antenna. To optimize the HF RFID system presented in Reiners et al. (2009) for use in a semicommercial growing-finishing stable, some alterations were made. Instead of incorporating the 
antenna into the feeding trough, a custom-made round HF RFID antenna (DTE Automation GmbH, Enger, Germany) was placed above the heads of the pigs as illustrated in Figure 2. This had the advantages of saving space at the feeding trough and possibly improved registrations of tags placed in the ear tags of the pigs. The theoretical detection range of the RFID antenna was $30 \mathrm{~cm}$. Each antenna was placed $46 \mathrm{~cm}$ above the ground to leave sufficient space between the trough and the antenna. Pigs occasionally chewed on the antenna, but the hard plastic housing protected the antenna from these bites. Extra cable protection with hard plastic tubes was added to protect the more vulnerable cables.

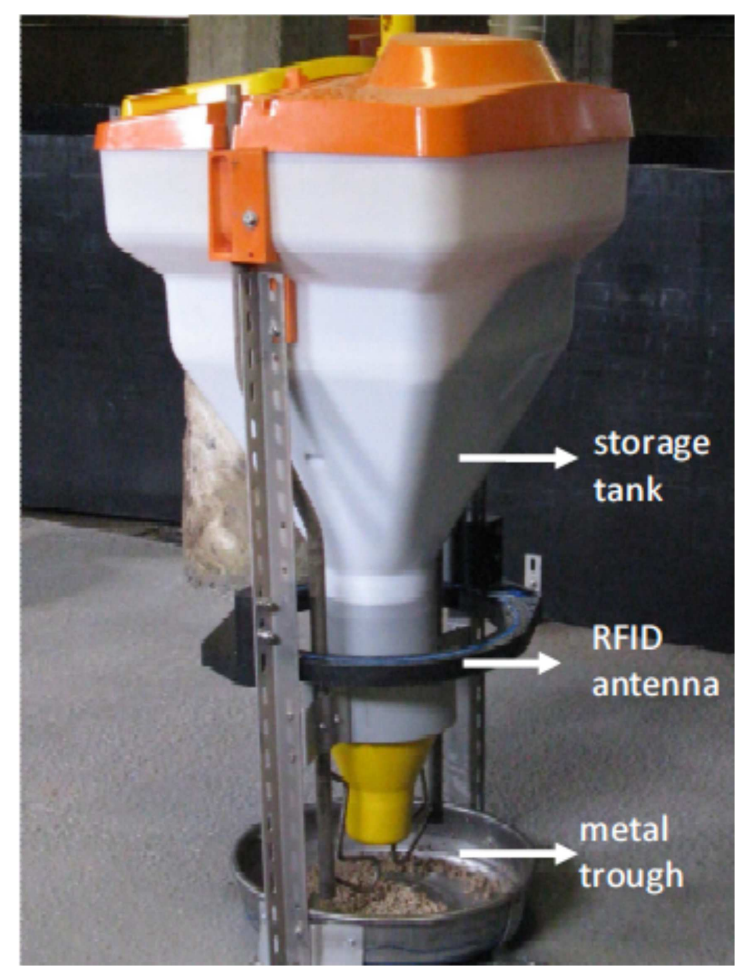

Figure 2: Pig feeder with a High Frequency RFID antenna attached around the storage tank above the metal trough.

In addition, multiplexers were used to connect multiple antennas to one reader. The 8 antennas were connected to one of two HF long range readers (ID ISC.LR2500-A, FEIG ELECTRONIC GmbH, Weilburg, Germany) with the use of two multiplexers (ID ISC.ANT.MUX-A, FEIG ELECTRONIC GmbH, Weilburg, Germany). This substantially reduced the cost of the system, but each antenna is only addressed once every cycle of the multiplexer. For a stationary tag in range of the antenna, the cycle time was $2 \pm 1 \mathrm{~s}$ (mean $\pm S D$ ). The cycle time depends on several factors, such as the number of tags in the read range and the anti-collision algorithms implemented in the HF system. This flexible and short cycle time allows switching rapidly between antennas and adjusting the cycle time to the circumstances. For example if one antenna has more tags in range than the other, more time will be given to this antenna. Movements of the pig during feeding can also move the tag in its ear in and out of range of the antenna. For all of these reasons, consecutive RFID registrations of a tag in a feeding pig's ear will display irregular time gaps between readings.

All the RFID registrations were continuously logged in a data file with three columns: 1) the timestamp of the registration to the second, 2) the unique code of the RFID tag and 3) the antenna where it was registered. 
VIDEO OBSERVATIONS

Video recordings were collected in the pen containing the pigs with two tags to observe the feeding visits of 20 focal pigs (10 barrows and 10 gilts) at one feeder in the pen. These 20 focal pigs were weighed, randomly selected (but so that sex and weight are equally distributed) and marked on day 44. Continuous observational analysis - to score pigs as feeding or not feeding - were performed on the video recordings of day 45 between 07:00 and 18:30 using The Observer 5.0 (Noldus Information Technology, Wageningen, The Netherlands). Day 45 was the first day of successful recording of the marked pigs. The observations were performed in the beginning of the fattening period, because small pigs move more during feeding and will be faster out of the range of the antenna due to their small size. This higher frequency and range of movement challenges the RFID system more than in the case of larger pigs. So, it can be seen as a 'worst case scenario'.

During the video observations, a pig was scored as feeding when it put its head in the trough and made a downward movement (deemed necessary to be able to take a mouthful of feed). The end of a visit was scored when the pig lifted its head, moved it away from the trough and showed the intention to also move its body away. A pig with its head downwards next to the trough was not considered to be feeding. During scoring, any other behaviour that could possibly influence the RFID registrations around the feed trough was noted. These video observations were used to calculate the number of feeding visits, the duration of individual feeding visits, the total feeding duration (during the observations) and the time between feeding visits for the focal pigs for the entire observation period.

\section{INSTANTANEOUS COMPARISON BETWEEN RFID AND VIDEO REGISTRATIONS}

Using video observations, continuous scoring of feeding pigs was performed. This yielded start and stop times of all feeding visits of the focal pigs down to the second. The RFID registrations were also logged to the second. Both the RFID and the video loggings occurred on the same computer, thus assuring time synchronization.

The RFID registrations were separated by time gaps of irregular length, whereas the video scoring was continuous. To compare these two, the continuous video scoring data were downsampled to instantaneous sampling observations with a sampling interval of $60 \mathrm{~s}$. For each sampling point (except the sampling point that coincides with the start of the observations) it was determined if the focal pig of interest was feeding or not during that second according to the video observations. Then, it was determined if there was minimum one RFID registration of that pig within a certain time window around the sampling point or not. The time windows used were of size 1, 3, 5, 7, 9, 11, 21, 31 and $51 \mathrm{~s}$ and were symmetrical around the video sampling second. For example, if the sampling point was the $60^{\text {th }}$ second of the observations and the time window was of size $5 \mathrm{~s}$, the time interval to look for RFID registrations ranged from second 58 to 62 . This procedure was repeated for every focal pig. 
COMPARISON OF VIDEO-BASED FEEDING DURATION AND RFID REGISTRATIONS

A general idea on the appropriateness of RFID technology to measure pigs' feeding patterns can be found by looking at the relationship between how many times a pig is detected by the RFID system during the observation time and how long it was observed as actually feeding according to the video recordings. Of all the video outcome variables, feeding duration is most likely to be correlated with number of RFID registrations. The number of registrations for each focal pig (sum of both ear tags) was regressed onto the feeding duration for that pig as determined from the video observations. The coefficient of determination $\mathrm{R}^{2}$ between both was calculated to quantify the correlation between these indicators for feeding patterns. Normality was checked with a Lilliefors test. Statistical significance was checked with an F-test of the overall fit. Statistical significance was considered at $P<0.05$. This analysis was performed using MATLAB R2010b (The MathWorks, Inc., Natick, Massachusetts, USA).

\section{PERFORMANCE OF THE RFID SYSTEM}

The sensitivity, specificity, accuracy and precision of the RFID system for detecting feeding pigs were calculated as follows:

$$
\begin{gathered}
\text { sensitivity }=\frac{T P}{P} \\
\text { specificity }=\frac{T N}{N} \\
\text { accuracy }=\frac{T P+T N}{P+N} \\
\text { precision }=\frac{T P}{T P+N-T N}
\end{gathered}
$$

$\mathrm{TP}=$ number of true positives = number of sampling points where the pig was feeding according to the video, with the corresponding time window containing at least one RFID registration;

$\mathrm{P}=$ number of positives $=$ number of sampling points where the pig was feeding according to the video;

$\mathrm{TN}=$ number of true negatives = number of sampling points where the pig was not feeding according to the video, with the corresponding time window containing no RFID registrations;

$\mathrm{N}=$ number of negatives $=$ number of sampling points where the pig was not feeding according to the video.

The sensitivity and specificity were calculated for each individual focal pig and for the total of all focal pigs for every time window. Accuracy and precision were calculated for the total of all focal pigs and for every time window. By calculating these measures using either two tags per pig or by looking at either the left- or the right-ear tag alone, a comparison between using 1 or 2 tags per pig was made. 
Also, the percentage of RFID registrations occurring during video feeding visits or within $10 \mathrm{~s}$ from a visit, the percentage of correctly identified feeding visits (thus the percentage of feeding visits on video during which an RFID registration occurred) and the agreement between RFID (for one or two tags per pig) and video on a per 20 second basis were calculated. Related to the latter, for every 20 second interval (2070 intervals in total) it was determined whether the RFID system considered the pig to be feeding (when the tag or one of the tags had a registration during the interval) and whether the video observations considered the pig to be feeding (during any part of the interval). These calculations were performed using MATLAB R2010b (The MathWorks, Inc., Natick, Massachusetts, USA).

\section{RESULTS}

\subsection{FEEDING PATTERN}

In Table 1 the results of weighing, video observations and RFID registrations for each focal pig are summarized. The average weight of the focal pigs on day 44 was $40.2 \pm 4.9 \mathrm{~kg}$ (mean \pm SD). In total $230 \mathrm{~h}$ were analysed on video ( $11.5 \mathrm{~h}$ for 20 focal pigs), giving $9.7 \mathrm{~h}$ of total feeding and 960 feeding visits captured on video. The feeding duration for the individual pigs during the $11.5 \mathrm{~h}$ of observations was $29 \pm 14$ min (mean \pm SD). The number of feeding visits was $48 \pm 28$ (mean \pm SD). The average duration of each pig's feeding visits was $42 \pm 18 \mathrm{~s}$ (mean $\pm \mathrm{SD}$ ) and the average gap between two feeding visits of the same pig was $16 \pm 13 \mathrm{~min}$ (mean \pm SD).

For the duration of feeding visits, one can see from Table 1 and the numbers above that the variance between pigs $(S D=18 \mathrm{~s}$ ) is much smaller than the variance within pigs (average of the $S D=56 \mathrm{~s}$ ). The same holds for the gaps between feeding visits, where the between-pigs SD is 13 min and the withinpigs average SD is $39 \mathrm{~min}$. This can be explained by the fact that some feeding visits are part of a single meal event (thus close to each other). 


\begin{tabular}{|c|c|c|c|c|c|c|c|c|c|c|c|}
\hline \multirow{3}{*}{$\begin{array}{l}\mathrm{nr} \text { focal } \\
\text { pig }\end{array}$} & \multicolumn{2}{|c|}{ WEIGHING DATA } & \multicolumn{6}{|c|}{ VIDEO OBSERVATIONS } & \multicolumn{3}{|c|}{ RFID REGISTRATIONS } \\
\hline & \multirow{2}{*}{$\begin{array}{l}\text { male/ } \\
\text { female }\end{array}$} & \multirow[t]{2}{*}{ weight $[\mathrm{kg}]$} & \multirow{2}{*}{$\begin{array}{c}\text { feeding } \\
\text { duration } \\
\text { [h:mm:ss] }\end{array}$} & \multirow{2}{*}{$\begin{array}{l}\text { \# feeding } \\
\text { visits }\end{array}$} & \multicolumn{2}{|c|}{$\begin{array}{l}\text { duration of feeding } \\
\text { visit [h:mm:ss] }\end{array}$} & \multicolumn{2}{|c|}{$\begin{array}{l}\text { gap between feeding } \\
\text { visits [h:mm:ss] }\end{array}$} & \multirow{2}{*}{$\begin{array}{c}\# \\
\text { registrations }\end{array}$} & \multirow{2}{*}{$\begin{array}{c}\% \\
\text { registrations } \\
\text { left tag }\end{array}$} & \multirow{2}{*}{$\begin{array}{c}\% \\
\text { registrations } \\
\text { right tag }\end{array}$} \\
\hline & & & & & average & stdev & average & stdev & & & \\
\hline 1 & male & 52 & $0: 21: 30$ & 16 & 0:01:21 & $0: 02: 25$ & $0: 42: 16$ & $0: 58: 54$ & 572 & 65 & 35 \\
\hline 2 & male & 45.9 & $0: 35: 45$ & 121 & $0: 00: 18$ & $0: 00: 18$ & 0:04:39 & $0: 16: 20$ & 653 & 28 & 72 \\
\hline 3 & male & 41.6 & $0: 19: 26$ & 32 & $0: 00: 36$ & $0: 00: 48$ & $0: 12: 19$ & $0: 30: 42$ & 464 & 59 & 41 \\
\hline 4 & male & 41.3 & $0: 15: 50$ & 20 & $0: 00: 48$ & $0: 00: 58$ & $0: 08: 52$ & $0: 27: 06$ & 463 & 58 & 42 \\
\hline 5 & male & 39.6 & 0:08:06 & 18 & $0: 00: 27$ & $0: 00: 52$ & $0: 16: 10$ & $0: 39: 48$ & 279 & 35 & 65 \\
\hline 6 & male & 38.8 & $0: 28: 40$ & 61 & $0: 00: 28$ & 0:00:41 & 0:09:44 & $0: 39: 54$ & 755 & 60 & 40 \\
\hline 7 & male & 38.4 & $0: 29: 50$ & 46 & 0:00:39 & 0:00:37 & $0: 12: 46$ & $0: 32: 12$ & 699 & 51 & 49 \\
\hline 8 & male & 37.3 & $0: 20: 18$ & 57 & $0: 00: 21$ & 0:00:20 & $0: 10: 17$ & $0: 27: 13$ & 656 & 55 & 45 \\
\hline 9 & male & 35.9 & 0:06:56 & 10 & 0:00:42 & 0:01:04 & $0: 58: 31$ & $2: 34: 31$ & 180 & 66 & 34 \\
\hline 10 & male & 35.7 & $0: 36: 15$ & 62 & $0: 00: 35$ & 0:00:41 & 0:09:27 & $0: 31: 12$ & 752 & 62 & 38 \\
\hline 11 & female & 45.4 & $0: 29: 31$ & 21 & $0: 01: 24$ & $0: 01: 56$ & $0: 26: 25$ & $0: 51: 09$ & 282 & 80 & 20 \\
\hline 12 & female & 44.5 & $0: 35: 11$ & 41 & $0: 00: 51$ & 0:01:09 & $0: 13: 36$ & $0: 36: 34$ & 751 & 58 & 42 \\
\hline 13 & female & 42.6 & $0: 49: 22$ & 55 & $0: 00: 54$ & 0:01:00 & $0: 11: 00$ & $0: 34: 59$ & 1101 & 50 & 50 \\
\hline 14 & female & 42.5 & $0: 35: 56$ & 65 & $0: 00: 33$ & $0: 00: 24$ & 0:09:41 & $0: 29: 03$ & 1405 & 64 & 36 \\
\hline 15 & female & 41.9 & $0: 32: 43$ & 34 & $0: 00: 58$ & $0: 01: 44$ & $0: 14: 11$ & $0: 44: 55$ & 833 & 32 & 68 \\
\hline 16 & female & 41.9 & $0: 31: 31$ & 72 & $0: 00: 26$ & $0: 00: 23$ & $0: 07: 52$ & $0: 20: 36$ & 874 & 54 & 46 \\
\hline 17 & female & 38.6 & $0: 15: 40$ & 16 & $0: 00: 59$ & $0: 01: 15$ & $0: 25: 55$ & $0: 49: 38$ & 345 & 69 & 31 \\
\hline 18 & female & 34.7 & $0: 21: 49$ & 51 & $0: 00: 26$ & $0: 00: 36$ & $0: 09: 32$ & $0: 22: 20$ & 534 & 36 & 64 \\
\hline 19 & female & 33.6 & 1:01:57 & 74 & 0:00:50 & $0: 00: 54$ & $0: 07: 31$ & $0: 22: 28$ & 840 & 34 & 66 \\
\hline 20 & female & 31 & 0:48:02 & 88 & 0:00:33 & 0:00:38 & 0:06:18 & $0: 22: 18$ & 1632 & 50 & 50 \\
\hline \multicolumn{2}{|c|}{ average values } & 40.2 & $0: 29: 13$ & 48 & $0: 00: 42$ & $0: 00: 56$ & $0: 15: 51$ & $0: 39: 36$ & 704 & 53 & 47 \\
\hline
\end{tabular}

Table 1: Results of weighing on day 44 and video observations and RFID registrations of 20 focal pigs (wearing 2 tags per pig) on day 45 of the fattening period from $7: 00$ to 18:30. Observations and registrations were for one feeder in a pen with 2 feeders. 


\subsection{COMPARISON VIDEO FEEDING DURATION AND RFID REGISTRATIONS}

In total 14070 RFID registrations of the focal pigs occurred during the observation time, with $704 \pm 363$ registrations per pig (mean \pm SD) (Table 1) on average. The scatter plot of the number of registrations per pig against the feeding duration as captured on video is illustrated in Figure 3 . If all pigs are considered, a coefficient of determination $\mathrm{R}^{2}$ of 0.53 is obtained. This low fit is mainly due to four pigs where the residual after linear regression is outside the $95 \%$ confidence interval (i.e., pigs $11,14,19$ and 20 , hereafter called 'outliers') - without those four pigs the $R^{2}$ increases to 0.88 . In both cases, overall fit was significant $(P<0.001)$.

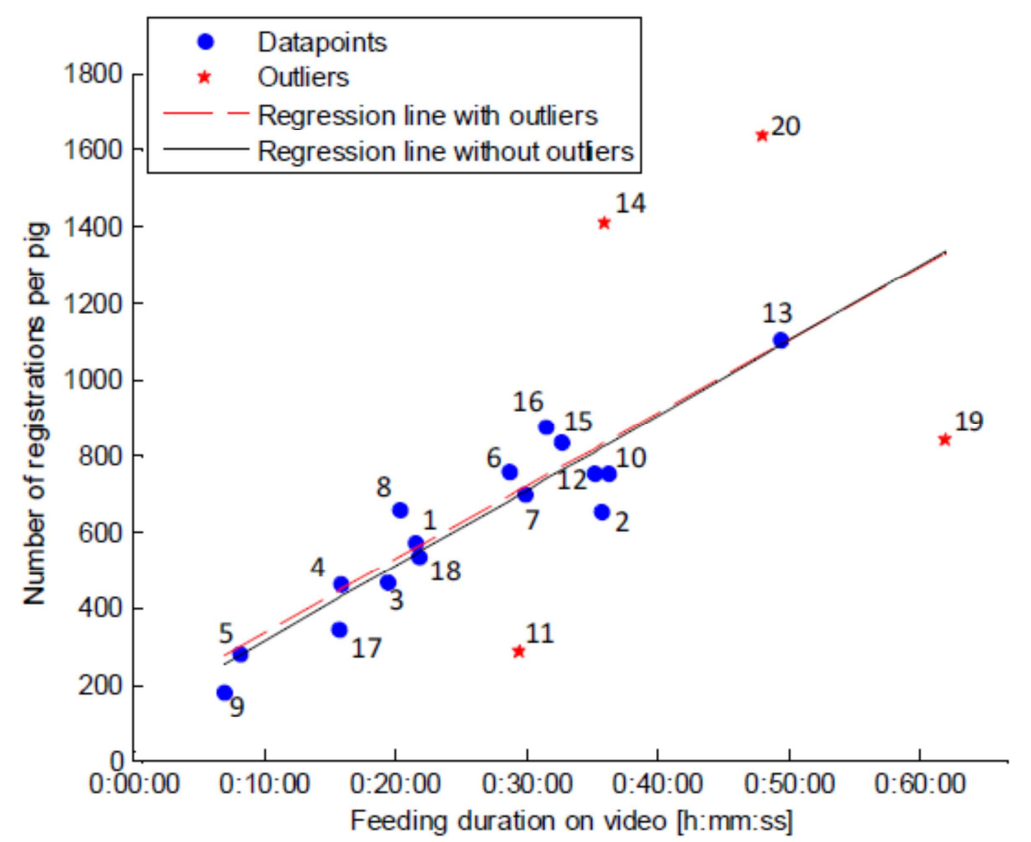

Figure 3: Linear regression of the number of RFID registrations per pig versus the feeding duration on video for each focal pig; with all pigs included $\left(y=143.14+0.32 x, R^{2}=0.5301\right)$; or with four outliers removed $\left(y=117.71+0.33 x, R^{2}=0.8795\right)$.

To illustrate the correspondence between the video based observations and RFID registrations for different pigs and to find an explanation for the outlier pigs, the signals registered for a 14 minute period containing feeding are illustrated in Figure 4 for pigs 11, 13 and 14 ( 2 outlier pigs and a pig on the regression line). It can be clearly seen that the RFID registrations during feeding for pig 11 are systematically lower than the video registrations, while those for pig 13 agree and those for pig 14 are higher. This explains why in Figure 3, pig 11 is below the regression line, pig 13 is on the line and pig 14 is above it. The lower RFID registrations for pig 11 and 19 (outliers on Figure 3) can be explained by these pigs' unusual habit of often lying down while feeding. These two pigs were the only focal pigs displaying this behaviour multiple times during the video observations. This prone position increased the distance between the RFID tags in their ears and the RFID antenna above their heads, which therefore may have reduced the number of registrations (Figure 4a). The pigs with a higher number of RFID observations compared to the video registrations of the feeding pattern (pig 14 and 20)(Figure 4c) can be explained by the two pigs' tendency to be in the vicinity of the RFID antenna without feeding. Pig 14 frequently lay down or walked next to the feeder without feeding, 
while pig 20, one of the lightest pigs in the pen, often stood close to the trough waiting for room to be able to feed, or was often chased away by other pigs while feeding.

All four of the outliers (pigs 11, 14, 19 and 20) showed different feeding behaviour than the rest of the focal pigs during the observation period. This explains their outlying value in the scatter plot (Figure 3). By examining the number of RFID registrations per day during the entire fattening period for pigs 11, 14, 19 and 20 compared to those of pig 13, we can deduce whether this feeding behaviour is consistent or not (data not shown). The number of registrations of pig 11 and pig 19 were typically lower than those of pig 13, while those of pig 14 and 20 were typically higher than those of pig 13. This was especially the case in the 30 days around the observation day.
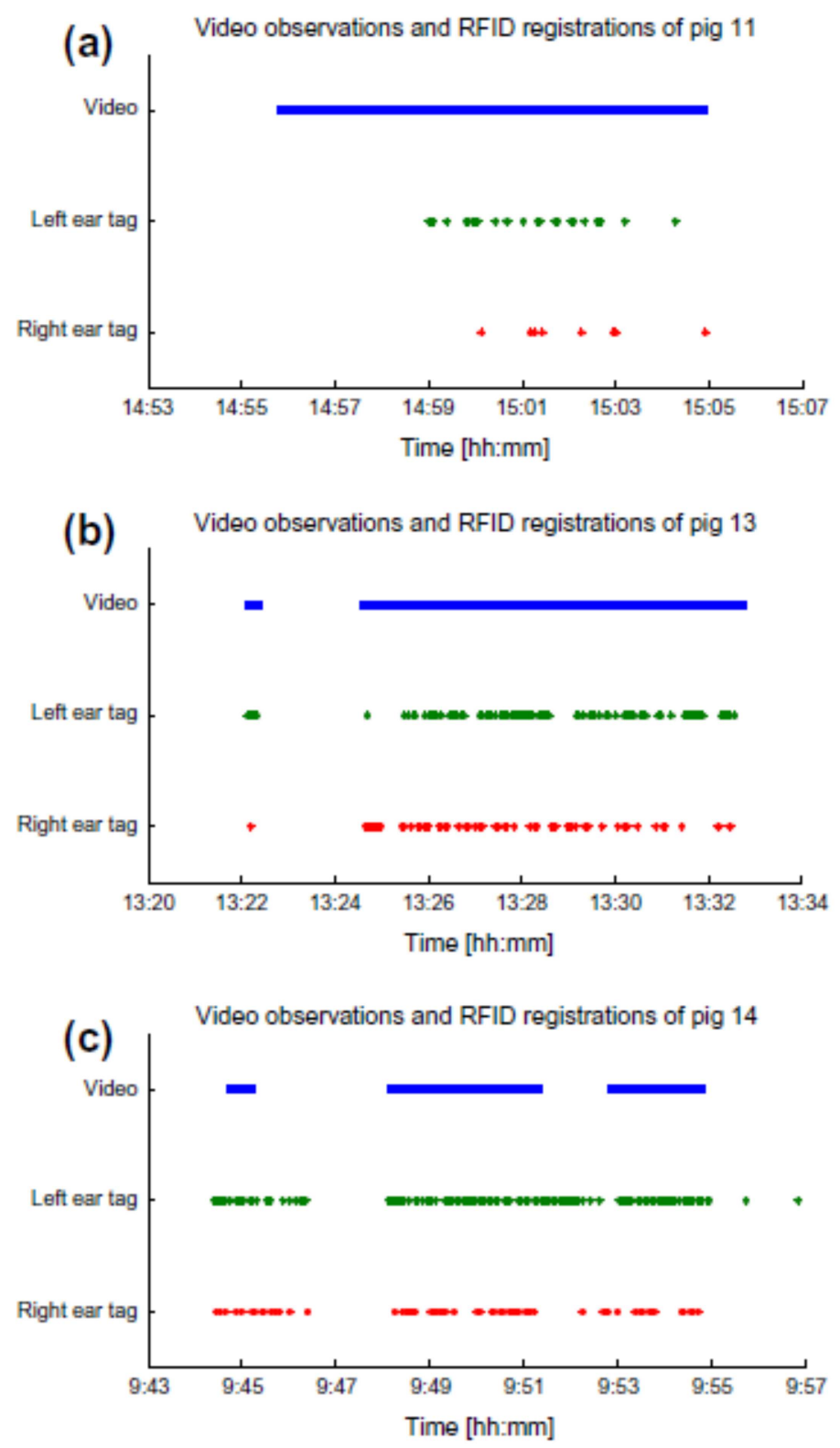

Figure 4: Video observations and RFID registrations of left tag and right tag of (a) pig 11, an outlier with a low ratio number of registrations versus feeding duration; (b) pig 13, a pig with an average ratio number of registrations versus feeding duration and; (c) pig 14, an outlier with a high ratio number of registrations versus feeding duration. 


\subsection{SENSITIVITY AND SPECIFICITY USING TWO TAGS PER PIG}

After transforming the continuous observations to instantaneous sampling observations with sampling period $60 \mathrm{~s}$ (see 0), 13800 sampling points were obtained for the 20 focal pigs (690 per pig). Of these video sampling points, 578 were moments where a pig was feeding. In Figure 5 the sensitivity and specificity calculated for all focal pigs are shown for different time window sizes around video sampling points used for the RFID registrations. Per pig, the number of sampling points where the pig was seen on video to be feeding was $29 \pm 13$ sampling points (mean \pm SD). A minimum specificity of $85 \%$ was always achieved, but this was not the case for individual pigs regarding sensitivity. With a time window of $7 \mathrm{~s}$, sensitivity of individual pigs varied between $43 \%$ and $100 \%$. For some pigs, a sensitivity of $85 \%$ was already reached for a time window of $3 \mathrm{~s}$, while for others this was only reached at a time window of $51 \mathrm{~s}$. This indicates that the different pigs had different time gaps between their RFID registrations during feeding. Pigs 2, 11 and 19 have a low sensitivity $(<70 \%)$ at a time window size of $7 \mathrm{~s}$; pig 11 and pig 19 were often seen lying down the feeder, which possibly caused erroneous registrations (see 4.2). Pig 2 was seen sitting down only once, but had many very short feeding visits (see also Table 1) that alternated with nosing on the ground, playing, etc. This relatively fast fluctuation of the RFID tags of pig 2 in and out of range of the RFID antenna probably also impaired registrations at several instants.

\subsection{SENSITIVITY AND SPECIFICITY USING ONE TAG PER PIG}

In Figure 6 the sensitivity of the RFID system for individual pigs using one tag per pig is shown for different time windows used for the RFID registrations. To achieve a minimum sensitivity of $85 \%$ a time window size of $31 \mathrm{~s}$ was needed for the average tag. Specificity was always larger than $85 \%$ (data not shown). Sensitivity of individual tags varied between $37 \%$ and $100 \%$ with a time window of $31 \mathrm{~s}$. For some tags a sensitivity of $85 \%$ was already reached for a time window of $5 \mathrm{~s}$, while for others this was not even reached at a time window of $51 \mathrm{~s}$. This indicates that the different tags had different time gaps between their RFID registrations during feeding. A large difference in performance between the left and right tag of the same pig could sometimes be observed. Technical performances of the tags, position and orientation of the tags during feeding and specific pig behaviours (e.g. preference for a certain side at the feeder) could possibly explain the differences in sensitivity of the two tags on the same pig. 

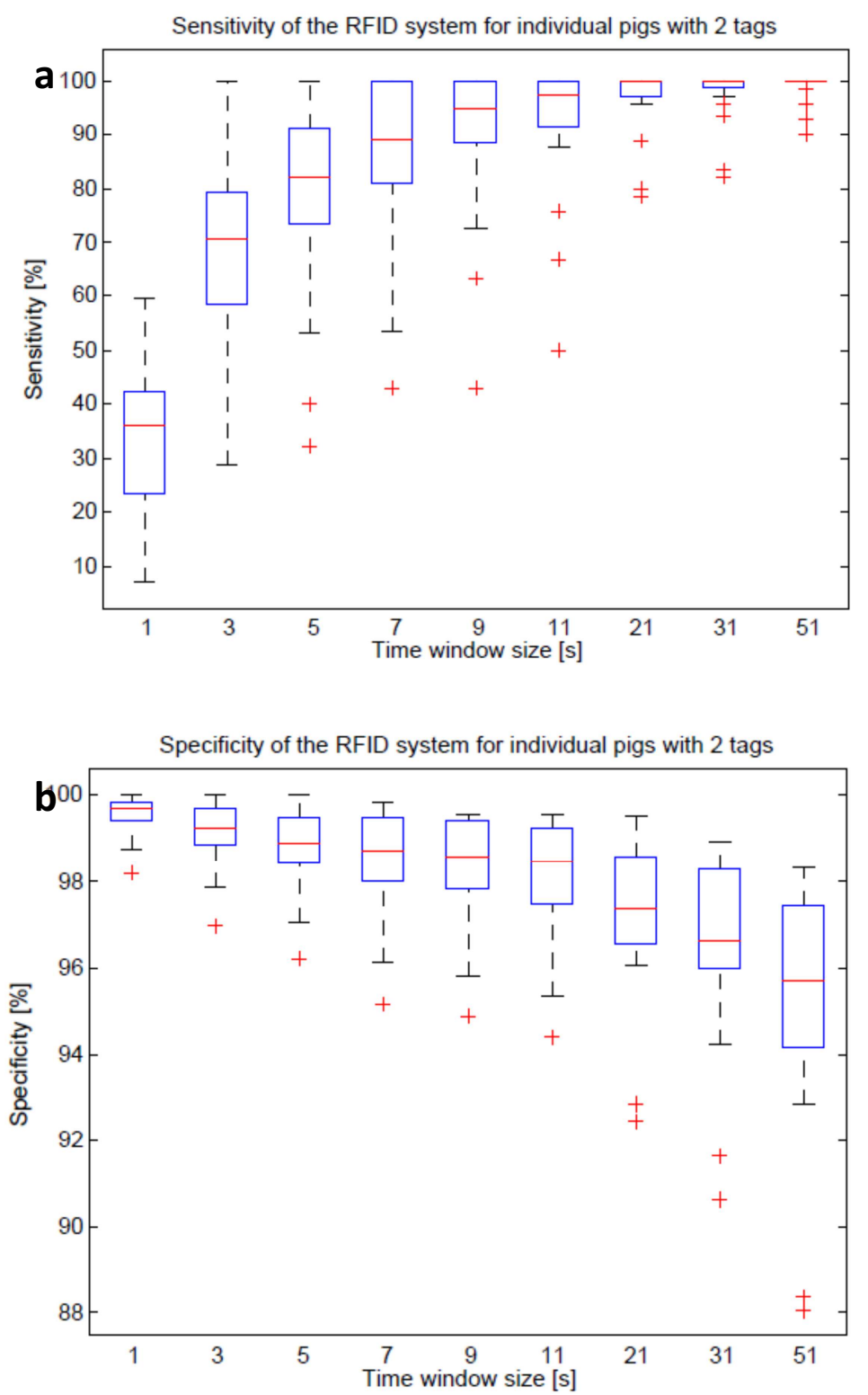

Figure 5: Sensitivity (a) and specificity (b) of the RFID system for individual pigs using two tags per pig versus the time window size used for the RFID registrations. On each box, the central mark is the median, the edges of the box are the 25th and 75th percentiles, the whiskers extend to the most extreme data points not considered outliers, and outliers are plotted individually. 


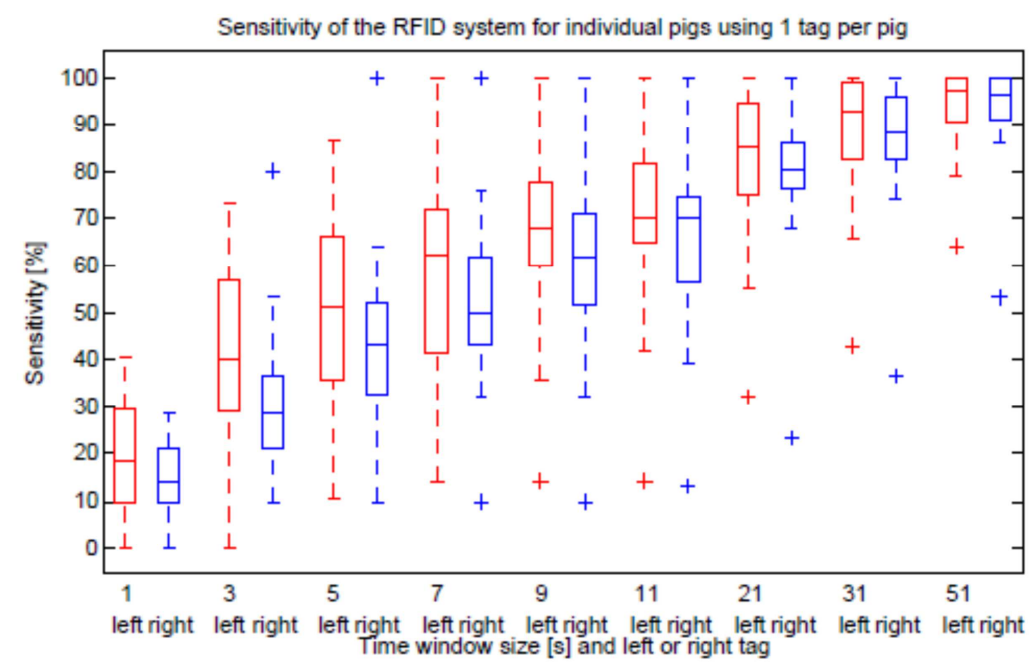

Figure 6: Sensitivity of the RFID system for individual pigs using one tag per pig versus the time window size used for the RFID registrations. On each box, the central mark is the median, the edges of the box are the 25th and 75th percentiles, the whiskers extend to the most extreme data points not considered outliers, and outliers are plotted individually.

\subsection{PERFORMANCE OF THE RFID SYSTEM}

In Figure 7 the sensitivity and specificity calculated for the RFID system using the data of all 20 focal pigs together is plotted against the time window size used for the RFID registrations. Examining the RFID registrations taken at the same moment as the video sampling point (time window size of $1 \mathrm{~s}$ ) gave a total sensitivity of $33.91 \%$ and specificity of $99.58 \%$ for pigs that had two tags. Increasing the time window to a size of $7 \mathrm{~s}$ increased the sensitivity to $83.22 \%$, while time window sizes of $21 \mathrm{~s}$ and $51 \mathrm{~s}$ gave sensitivities of $96.02 \%$ and $98.62 \%$, respectively (all sensitivities are for two tags per pig). When the window size increased from $7 \mathrm{~s}$ to $21 \mathrm{~s}$ and $51 \mathrm{~s}$, the specificity for the RFID system using two tags per pig decreased from $98.50 \%$ to $97.28 \%$ and $95.23 \%$, respectively. The sensitivity and specificity of the total system using either the left ear tags or the right ear tags is also shown in Figure 7.

The difference in performance between either left or right tags is small (Figure 7), but there is a difference in performance when using one versus two tags per pig. The sensitivity when using two tags per pig is higher than that of using one tag per pig. In contrast, two tags per pig resulted in slightly lower specificity. It is indeed logical that two tags give more registrations than one tag, leading to a higher rate of true positives (RFID registrations while feeding) but also a slightly higher rate of false positives (RFID registrations while no feeding occurs).

A receiver (or relative) operating characteristic (ROC) curve is shown for the RFID system for all 20 focal pigs together, using either the left tags, the right tags, or two tags per pig (Figure 8). An ROC curve is a plot of the sensitivity (or the true positive rate) versus one minus the specificity (or the false positive rate). The performance is generally lower when using one tag per pig versus using two tags per pig (Figure 8). Sensitivity is above $85 \%$ for one tag per pig at a time window of size $31 \mathrm{~s}$. This point corresponds to a sensitivity of $87.02 \%$ and specificity of $97.49 \%$ for the left tags and a sensitivity of $85.99 \%$ and specificity of $97.59 \%$ for the right tags. For achieving a minimum sensitivity 
of $85 \%$, a time window of $9 \mathrm{~s}$ suffices for two tags per pig. For a time window of size $9 \mathrm{~s}$, the sensitivity and specificity using two tags per pig are $88.58 \%$ and $98.34 \%$, respectively.

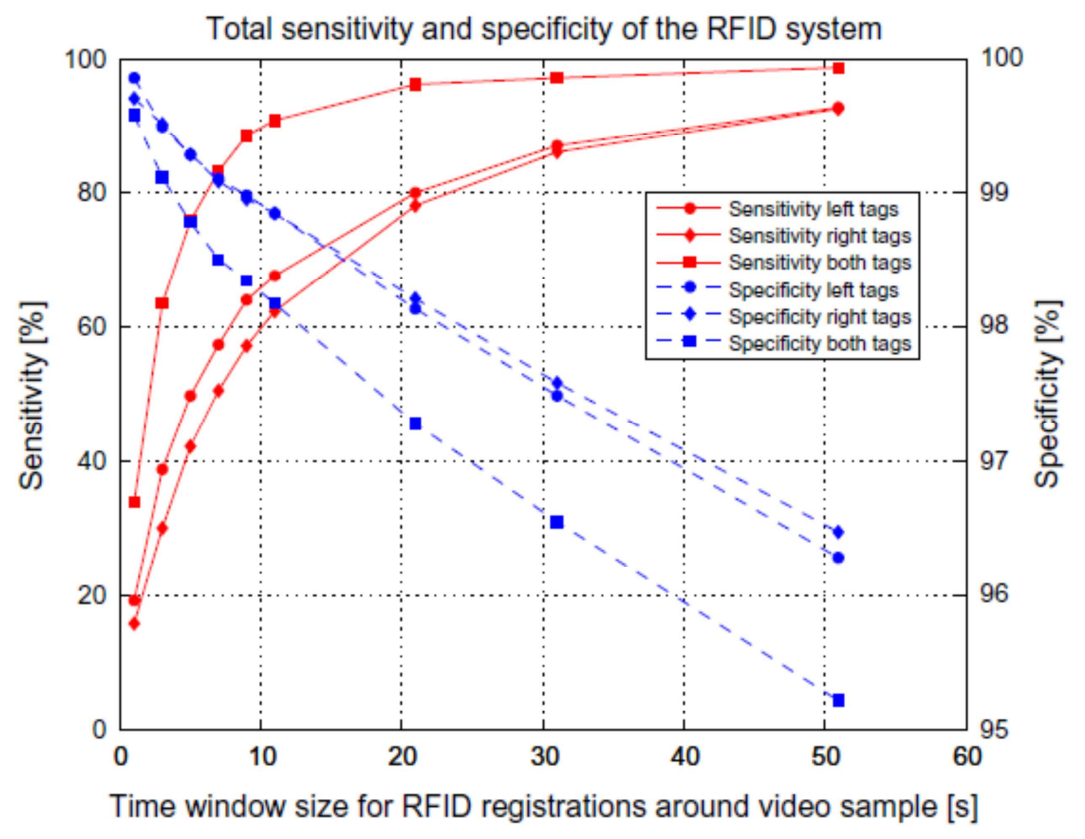

Figure 7: Total sensitivity and specificity of the RFID system using one or two tags per pig versus the time window size used for the RFID registrations.

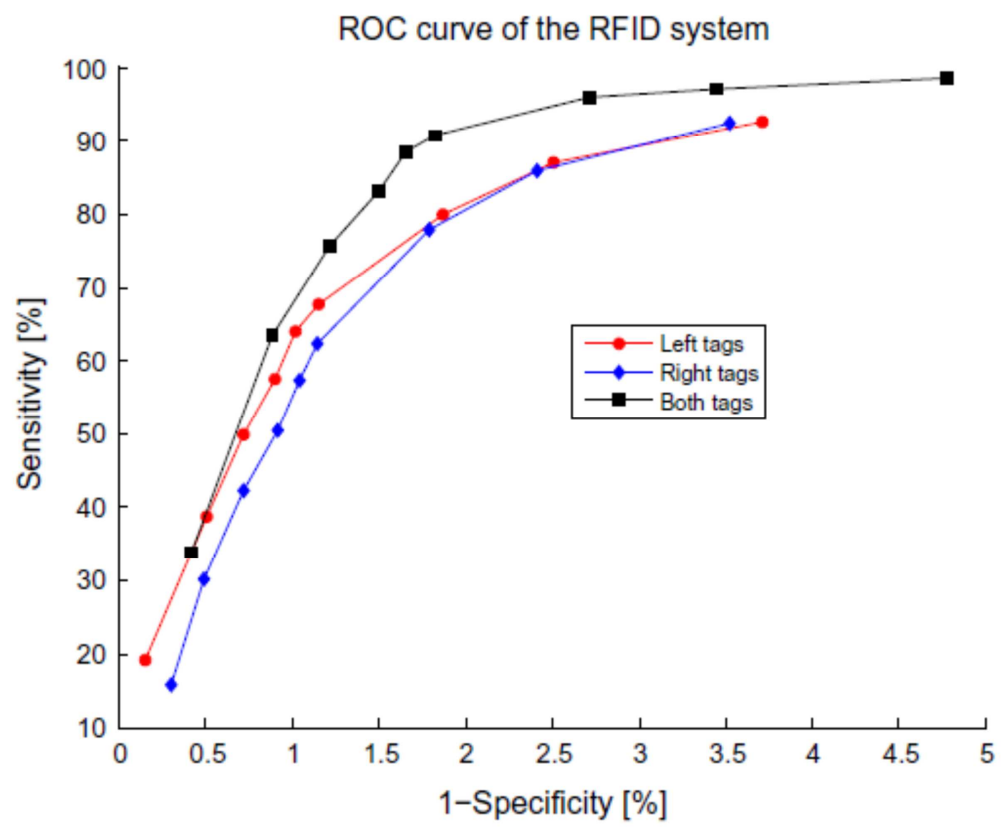

Figure 8: Receiver operating characteristic (ROC) curve of the RFID system, with the markers representing the time window sizes used $(1,3,5,7,9,11,21,31$ and $51 \mathrm{~s}$; in this order from left to right).

Total accuracy and precision of the system can be calculated using the formulas in section 0 and is shown in Figure 9. Accuracy is always very high (more than $95 \%$ ), thus the proportion of true results of the RFID system is very high. However, the precision, the proportion of true positives against all the positive results, varies between time windows used. For 2 tags per pig, the highest precision is 
$77.78 \%$ for time window size $1 \mathrm{~s}$ and it gradually decreases to $47.46 \%$ for time window size $51 \mathrm{~s}$. Indeed, when an RFID registration occurs the exact same second we look at the video sample, the precision is higher than when we find an RFID registration in a $51 \mathrm{~s}$ time window and then look at a $1 \mathrm{~s}$ duration video sample in the middle of the time window. At the suggested time window size of $9 \mathrm{~s}$, the precision is $70.04 \%$, so only $70 \%$ of the positive signals reflect that the pig is eating. This corresponds with an error rate of the RFID system (using 2 tags, $9 \mathrm{~s}$ time window) of $29.96 \%$. From Figure 9 we can also conclude that using 2 tags instead of 1 tag does not necessarily increase the precision of the system.

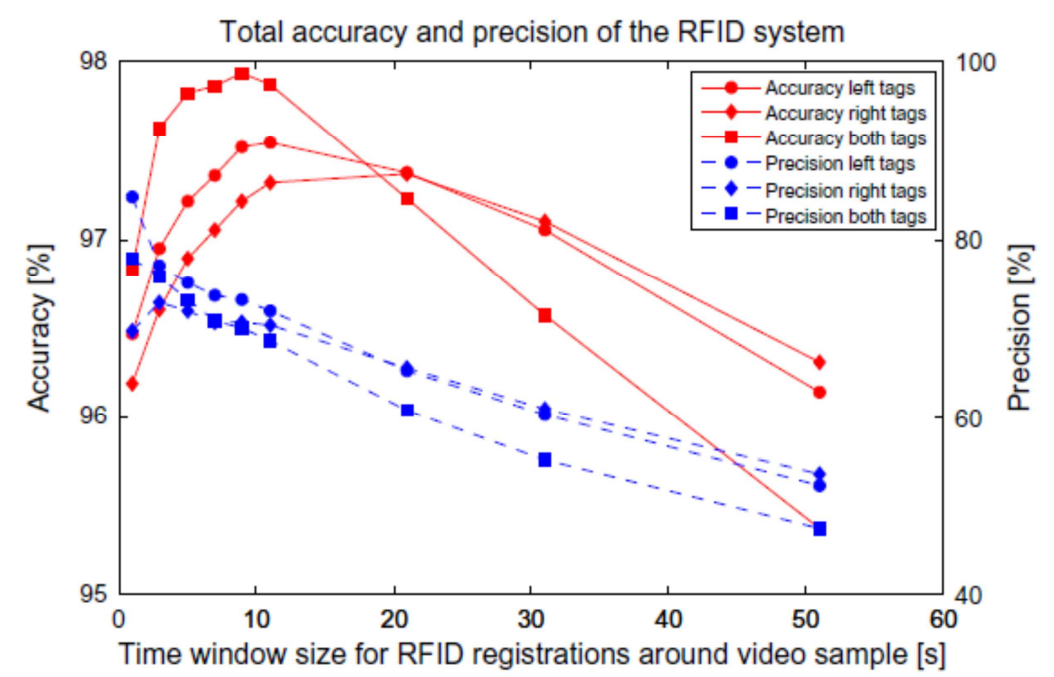

Figure 9: Total accuracy and precision of the RFID system using one or two tags per pig versus the time window size used for the RFID registrations.

The percentage of RFID registrations occurring during a video feeding visit was on average $76.13 \%$ with a SD of 14.51 percentage points (pp). The total percentage of RFID registrations during video feeding was $77.11 \%$. A very low percentage of correct RFID registrations was observed for pig 2, namely $25.42 \%$, which might be explained by this pig's fast alternation between feeding and not feeding (see Table 1). This pig had short gaps between feeding and a high number of very short feeding visits. During the observations it was noted that pig 2 very often paused its feeding visits for exploring the feeder construction or the ground. This was scored as not feeding, but RFID registrations probably continued during these pauses. The percentage of RFID registrations during or within $10 \mathrm{~s}$ from a video feeding visit was considerably higher, on average $91.04 \%$ with a SD of $7.47 \mathrm{pp}$ for the individual pigs and in total $92.23 \%$.

The percentage of video feeding visits containing an RFID registration of either of the 2 tags of the pig was on average $91.40 \%$ with a SD of $8.09 \mathrm{pp}$. The total percentage of correctly identified video feeding visits was only $89.17 \%$, mainly due to the high number of very short feeding visits of pig 2 . For this pig, the percentage of correctly identified visits was only $65.29 \%$, while for the other pigs this was always above $82 \%$. The percentage of video feeding visits containing an RFID registration of the left tag of the pigs was on average $76.04 \%$ with a SD of $15.15 \mathrm{pp}$, while for the right tags this was $71.90 \%$ with a SD of $13.52 \mathrm{pp}$. 
The agreement between video and RFID on a per 20 second basis, using 2 tags per pig, was on average $98.47 \%$ with a SD of $1.05 \mathrm{pp}$. Using one tag per pig, the agreement was on average $97.87 \%$ with a SD of $1.67 \mathrm{pp}$ or $97.88 \%$ with a SD of $1.32 \mathrm{pp}$, for left or right tags respectively.

\section{DISCUSSION}

High variability in the feeding patterns of individual, healthy pigs was found (Table 1 ) and is in agreement with the observations of other researchers (Brown-Brandl et al., 2013; Hessel and Van den Weghe, 2011).

For the HF RFID system, using two tags per pig, a sensitivity of $88.58 \%$ and a specificity of $98.34 \%$ could be achieved for a time window of $9 \mathrm{~s}$. These performance values were based on a minimal sensitivity of $85 \%$, but with the ROC curve in Figure 8 the reader can analyse the sensitivity and specificity of the system using different time windows.

The achieved values illustrate that the HF RFID system is capable of accurately registering feeding pigs at an industrial-commercial feeder in a commercial-like pig barn. These results correspond to those found in literature, although the validation methods used were different. Reiners et al. (2009) reported an identification rate of $97.3 \%$ for a similar HF RFID system with the antenna incorporated in the trough and no multiplexer. This identification rate corresponds to the percentage of trough visits detected by both RFID and video measurements. They also reported that in $64 \%$ of the cases, the piglets were identified with a slight time delay between RFID and video. Brown-Brandl et al. (2013) developed and validated an LF RFID system for monitoring feeding behaviour of cattle and swine and mention that the cattle system was in agreement with the video $98.3 \%$ of the time, and the swine system was in agreement $98.7 \%$ of the time. This agreement corresponds to the percentage of $20 \mathrm{~s}$ (swine system) or $30 \mathrm{~s}$ (cattle system) intervals where video and RFID system were identical (either both feeding or both not feeding).

In terms of validation of these RFID systems, the results obtained in this study illustrate the need to calculate sensitivity (likelihood that a feeding pig is detected by the RFID system) and specificity (likelihood that a pig that is not feeding is not detected by the RFID system) separately. Accuracy (likelihood that a pig's status is correctly identified by the RFID system) and agreement (on a per $20 \mathrm{~s}$ basis) gave very high values, but might not provide a complete representation of the performance of the system. Due to the low prevalence of feeding, the correct negatives contributed much more to the accuracy and agreement percentage than the correct positives.

It should be noted that the fact that the specificity was always very high compared to the sensitivity can be partially explained by the high number of samples ( $N=13222$ out of 13800 samples in total) where no feeding was observed on the video (Figure 5, Figure 7). This makes that accidental registrations only have a small influence on the specificity percentage. The feeding duration of a pig accounts for only a small amount of time during the day (Table 1), therefore this specificity is a good representation for the performance of the system throughout the day.

On the other hand, the sensitivity depended highly on the situation. The size of the time window over which the RFID registrations are compared with the video observations had a large influence on the sensitivity (Figure 7). This can be explained by the fact that the multiplexer creates time gaps between the RFID registrations, which are found to be larger when registering tags attached to pigs 
than for a stationary tag that remains continuously in range of the antenna. In addition, these gaps are not constant, but depend on the number of tags in range of the antenna and the behaviour of the pig. A feeding pig moves during feeding, for example picks up food from different places in the feeder trough, lifts its head to look around while chewing. This leads to changes in the position and orientation of the RFID tag with respect to the antenna. If those positional changes during feeding take the tag into and out of the detection range of the antenna, the time between RFID registrations of this pig will increase accordingly. These gaps between registrations can be dealt with by defining a visit criterion to cluster RFID registrations into feeding visits (Mendes et al., 2011). From the observed results it is recommended to use a visit criterion of minimum $9 \mathrm{~s}$ for this system when using two tags per pig, because the system performance was suboptimal for time window sizes smaller than $9 \mathrm{~s}$ (Figure 8).

It should be noted that the age of the pigs and their size might also have an impact on the sensitivity of this system. Small pigs are further away from the antenna than large pigs and can also be more active during feeding. We can expect the performance of the system to be lowest for small pigs, but further work is needed to confirm this. Also the effect of time window size on the performance of the system might be different for different ages of pigs, and hence may lead to a different optimal visit criterion.

We found that the performance of the RFID system is different when using one versus two tags per pig. Sensitivity is higher, but specificity is lower when using two instead of one tag per pig (Figure 7). When using two tags the overall performance (accounting for both sensitivity and specificity) is higher (Figure 8). Two tags per pig, however, do imply a higher cost and time investment (for placing and removing the tags). To make a decision on the number of tags to use per pig (one or two), more information is needed. The accuracy of the feeding patterns that can be extracted from the RFID data and the accuracy of possible applications such as early detection of problems could be influenced by the number of tags. Therefore, in future experiments (some) pigs will still be equipped with two tags.

It was also observed that the sensitivity can depend on the tag or the ear of the pig (Figure 6). As pigs may have a favourite feeding position or orientation at the trough one of both ears may be more frequently in the range of the antenna. This could be a valid explanation for the registration differences between both tags for one pig, but no clear cases of such behaviours were noted during the video observations. Another explanation for this observation could be variation in the technical performance of the tags (e.g. a different minimal activation field), but this cannot be confirmed based on the results obtained in this study. Although the RFID antenna was reported to have a theoretical range of $30 \mathrm{~cm}$, this range depends highly on the position and orientation of the tags (Finkenzeller, 2010). To know exactly where tags are registered and to know the range of the RFID system in practice, range measurements should be made, including several possible positions and orientations of the tags. Also the factors possibly influencing the registrations such as tag and antenna should be examined. These measurements will provide a better insight into the functioning and limitations of the RFID system.

The results also indicate that the RFID registrations, and hence the sensitivity, are influenced by behaviour of the individual pig. While a good correlation $\left(R^{2}=0.88\right)$ between the number of registrations and the feeding duration was observed for most pigs (Figure 3), there were 4 outliers, which could be explained based on the specific behaviour of these pigs. Mainly due to these 4 pigs, 
the overall correlation between number of registrations and feeding duration was low $\left(R^{2}=0.53\right)$. Ranking order, specific feeding behaviour (lying down, etc.) and interactions between pigs influence the RFID registrations and the sensitivity and specificity of the system for individual pigs (Figure 5). Further deduction - and validation using video observations as a golden standard - of feeding pattern variables such as visits and meals from the RFID data is needed to determine whether the feeding pattern of every pig can be estimated based on RFID data alone.

When using feeding patterns to detect possible health, welfare and performance problems, the feeding patterns for individual pigs must be analysed and each pig should be its own reference due to the variability found. For disease detection it might be more interesting to detect changes in individual pigs' feeding patterns through time instead of detecting pigs that have a different feeding pattern than their pen mates. In that sense, it is also possible that the outlier pigs displaying other behaviours at the trough (such as lying down while feeding or standing next to the trough without feeding) - and hence also a bad correlation between RFID registrations and feeding duration, or a low performance of the RFID system - would not form an issue for problem detection if this behaviour stays constant throughout time (see 4.2). Further research is, however, needed to examine feeding patterns and RFID performances in measuring feeding patterns through time.

For a system designed to detect feeding pigs, it was also important to know the precision (likelihood that a pig detected by the RFID system is actually feeding). In the analysis of this study instantaneous video samples of $1 \mathrm{~s}$ duration were compared with RFID registrations during a time window. In case of the precision, the values achieved with time window sizes larger than $1 \mathrm{~s}$ were not representing the performance of the system since it is not clear whether the pig is feeding or not during the rest of the time window not spanned by the video sample of $1 \mathrm{~s}$ duration. A better representation is the percentage of RFID registrations during video visits, which not surprisingly was about the same as the precision for a $1 \mathrm{~s}$ time window.

The percentage of RFID registrations outside video visits, $23.87 \%$, is a correct measure of the error rate of the system. Such accidental registrations occur when a pig is detected while passing the feeder or registered before or after a feeding visit while it is approaching the feeder trough, standing next to the feeder waiting for space at the trough or when it is nosing on the ground, for example. Many of these false positives were close to actual feeding visits. It might be doubted whether the observer correctly identified start and stop time of every visit and whether the definition of a feeding visit is $100 \%$ accurate (a pig nosing on the ground might be feeding on spilled food for example). Again, further deduction of visits and meals will clarify if it is possible to measure the feeding patterns of individual pigs and lower the error rate. Also, for disease detection these false positives might not be an issue, since it is expected that an ill pig will struggle to get close enough to the feeder and will not often be (falsely) registered.

\section{CONCLUSION}

The HF RFID system developed in this study can be used to measure feeding patterns of growingfinishing pigs in a commercial or simulated commercial situation. A correlation between number of RFID registrations and feeding duration was found, but specific behaviours such as lying down while feeding or standing next to the trough without accessing the feed might impair this correlation. Overall performance of the RFID system is dependent on the time window sizes used to compare 
with video observations and varies with pig, tag and the number of tags per pig. Many of the RFID registrations that occurred outside of feeding visits were close to actual feeding visits. With an achievable a sensitivity of $88.58 \%$ and a specificity of $98.34 \%$, the HF RFID system performed well in registering the feeding visits of the pigs.

The system presented here has good potential for measuring feeding patterns of individual growingfinishing pigs in commercial pig houses, for research purposes, or for detecting latent problems with pigs. For defining feeding pattern variables, such as visits and meals, further work is needed to find the optimal criteria to cluster the RFID registrations. For detecting health, welfare and production problems, however, monitoring of the raw RFID data as well as monitoring visits and meals might be possible.

\section{ACKNOWLEDGMENTS}

Jarissa Maselyne is funded by a PhD grant of the Agency for Innovation by Science of Technology (IWT Flanders - project SB 111447). The results presented are obtained in the framework of the ICTAGRI era-net project entitled PIGWISE "Optimizing performance and welfare of fattening pigs using High Frequent Radio Frequency Identification (HF RFID) and synergistic control on individual level" (Call for transnational research projects 2010). Special thanks go to the technical staff of the ILVO for the work and technical support provided during the course of the project and to Miriam Levenson for the English-language corrections.

\section{REFERENCES}

Ahrendt, P., Gregersen, T., and Karstoft, H., 2011. Development of a real-time computer vision system for tracking loose-housed pigs. Computers and Electronics in Agriculture 76, 169-174.

Artmann, R., 1999. Electronic identification systems: state of the art and their further development. Computers and Electronics in Agriculture 24, 5-26.

Brown-Brandl, T.M. and Eigenberg, R.A., 2011. Development of A Livestock Feeding Behavior Monitoring System. Transactions of the Asabe 54, 1913-1920.

Brown-Brandl, T.M., Rohrer, G.A., and Eigenberg, R.A., 2013. Analysis of feeding behavior of group housed growing-finishing pigs. Computers and Electronics in Agriculture 96, 246-252.

Bruininx, E.M.A.M., van der Peet-Schwering, C., Schrama, J.W., den Hartog, L.A., Everts, H., and Beynen, A.C., 2001a. The IVOG (R) feeding station: a tool for monitoring the individual feed intake of group-housed weanling pigs. Journal of Animal Physiology and Animal NutritionZeitschrift fur Tierphysiologie Tierernahrung und Futtermittelkunde 85, 81-87.

Bruininx, E.M.A.M., van der Peet-Schwering, C., Schrama, J.W., Vereijken, P.F.G., Vesseur, P.C., Everts, H., den Hartog, L.A., and Beynen, A.C., 2001b. Individually measured feed intake characteristics and growth performance of group-housed weanling pigs: Effects of sex, initial body weight, and body weight distribution within groups. Journal of Animal Science 79, 301-308.

Chapinal, N., Veira, D.M., Weary, D.M., and von Keyserlingk, M.A.G., 2007. Technical note: Validation of a system for monitoring individual feeding and drinking behavior and intake in group-housed cattle. Journal of Dairy Science 90, 5732-5736.

Chawla, V. and Ha, D.S., 2007. An overview of passive RFID. IEEE Communications Magazine 11-17.

Cornou, C., Vinther, J., and Kristensen, A.R., 2008. Automatic detection of oestrus and health disorders using data from electronic sow feeders. Livestock Science 118, 262-271. 
DeVries, T.J., von Keyserlingk, M.A.G., Weary, D.M., and Beauchemin, K.A., 2003. Technical note: Validation of a system for monitoring feeding behavior of dairy cows. Journal of Dairy Science 86, 3571-3574.

Eradus, W.J. and Jansen, M.B., 1999. Animal identification and monitoring. Computers and Electronics in Agriculture 24, 91-98.

Finkenzeller, K., 2010. RFID Handbook: Fundamentals and Applications in Contactless Smart Cards, Radio Frequency Identification and Near-Field Communication third ed. John Wiley \& Sons Ltd, West Sussex, United Kingdom.

Frost, A.R., Schofield, C.P., Beaulah, S.A., Mottram, T.T., Lines, J.A., and Wathes, C.M., 1997. A review of livestock monitoring and the need for integrated systems. Computers and Electronics in Agriculture 17, 139-159.

Gonyou, H.W. and Lou, Z., 2000. Effects of eating space and availability of water in feeders on productivity and eating behavior of grower/finisher pigs. Journal of Animal Science 78, 865-870.

Hart, B.L., 1988. Biological basis of the behavior of sick animals. Neuroscience \& Biobehavioral Reviews 12, $123-137$.

Hessel, E.F. and Van den Weghe, H.F.A., 2011 Individual online-monitoring of feeding frequency and feeding duration of group-housed weaned piglets via high frequent radiofrequency identification (HF RFID). in: Lokhorst C. and Berckmans D. (Eds.), European Conference on Precision Livestock Farming, pp. 210-222.

Hyun, Y. and Ellis, M., 2002. Effect of group size and feeder type on growth performance and feeding patterns in finishing pigs. Journal of Animal Science 80, 568-574.

Hyun, Y., Ellis, M., McKeith, F.K., and Wilson, E.R., 1997. Feed intake pattern of group-housed growing-finishing pigs monitored using a computerized feed intake recording system. Journal of Animal Science 75, 1443-1451.

Lind, N.M., Vinther, M., Hemmingsen, R.P., and Hansen, A.K., 2005. Validation of a digital video tracking system for recording pig locomotor behaviour. Journal of Neuroscience Methods 143, 123-132.

Mendes, E.D.M., Carstens, G.E., Tedeschi, L.O., Pinchak, W.E., and Friend, T.H., 2011. Validation of a system for monitoring feeding behavior in beef cattle. Journal of Animal Science 89, 2904-2910.

Nielsen, B.L., Lawrence, A.B., and Whittemore, C.T., 1996. Feeding behaviour of growing pigs using single or multi-space feeders. Applied Animal Behaviour Science 47, 235-246.

Reiners, K., Hegger, A., Hessel, E.F., Bock, S., Wendl, G., and Van den Weghe, H.F.A., 2009. Application of RFID technology using passive HF transponders for the individual identification of weaned piglets at the feed trough. Computers and Electronics in Agriculture 68, $178-184$.

Ruiz-Garcia, L. and Lunadei, L., 2011. The role of RFID in agriculture: Applications, limitations and challenges. Computers and Electronics in Agriculture 79, 42-50.

Schwartzkopf-Genswein, K.S., Huisma, C., and McAllister, T.A., 1999. Validation of a radio frequency identification system for monitoring the feeding patterns of feedlot cattle. Livestock Production Science 60, 27-31.

Wathes, C.M., Kristensen, H.H., Aerts, J.M., and Berckmans, D., 2008. Is precision livestock farming an engineer's daydream or nightmare, an animal's friend or foe, and a farmer's panacea or pitfall? Computers and Electronics in Agriculture 64, 2-10.

Weary, D.M., Huzzey, J.M., and von Keyserlingk, M.A.G., 2009. BOARD-INVITED REVIEW: Using behavior to predict and identify ill health in animals. Journal of Animal Science 87, 770-777. 